ISSN 0103-9954

\title{
MODELACIÓN DEL EFECTO DE LA PODA Y RALEO SOBRE LAS DEFORMACIONES RESIDUALES LONGITUDINALES EN Eucalyptus globulus Labill.
}

\author{
MODELING THE EFFECT OF PRUNING AND THINNING ON GROWTH STRAIN OF \\ Eucalyptus globulus Labill.
}

\author{
Eduardo Navarrete ${ }^{1}$ Carolina Valenzuela ${ }^{2}$ Leif Nutto ${ }^{3}$
}

\begin{abstract}
RESUMEN
Se estudió una plantación de Eucalyptus globulus ubicada en el sector precordillerano andino de la región del Bío Bío, Chile, con el objetivo de evaluar y modelar el efecto de la poda y raleo sobre las deformaciones residuales longitudinales (DRL). El lugar de estudio correspondió a un rodal de 12 años de edad, el cual fue intervenido a los 2 años y medio de establecido, donde se evaluaron distintas intensidades de poda ( $0 \%$ y $60 \%$ de la altura total) y densidades de raleo $\left(1.600\right.$ árb $\cdot h^{-1}\left(\sin\right.$ raleo) y 800 árb $\left.\cdot h^{-1}\right)$. En cada tratamiento definido por la combinación de los dos niveles de poda y raleo, se estudió además, el efecto de la clase de copa (dominante, codominante e intermedia) sobre el comportamiento de las DRL. En cada unidad de muestreo se registraron variables a nivel de árbol individual, estimando los parámetros de rodal más relevantes. Se muestreó un total de 36 árboles, midiéndose en cada uno de ellos las DRL a nivel del Dap con un extensómetro diseñado por el CIRAD-Forêt. Los resultados determinaron que la poda y raleo no afectaron significativamente el comportamiento de las DRL en Eucalyptus globulus; en cambio, la clase de copa, tuvo un efecto altamente significativo sobre la variación de las deformaciones residuales, presentando los árboles dominantes mayores niveles de deformación que las clases de copa codominante e intermedia, respectivamente. El modelo de estimación incluyó como variables predictivas del comportamiento de las DRL, las expresiones logarítmicas del Dap y del inverso multiplicativo de la varianza de los radios de copa, además de la poda, presentando un $\mathrm{R}^{2}$ de 0,44 y un EEE de 0,132 .
\end{abstract}

Palabras clave: Eucalyptus; clase de copa; manejo silvícola; deformación residual longitudinal.

\begin{abstract}
A 12-year-old Eucalyptus globulus plantation forest was studied with the aim of assessing and modeling the effect of both pruning and thinning activities on the growth strain. The stand is located in the Andean foothills of Bío Bío region in Chile. Two forest management activities were carried out after 2 and half years from planting, and were evaluated according to different pruning intensities (from 0 to $60 \%$ from total height of the tree) and different thinning densities (800 UPH with thinning and $1.600 \mathrm{UPH}$ without thinning). Furthermore, the effect of the crown classes (dominant, co-dominant and intermediate crown) was studied by the combination of two treatments on the behavior of the growth strain. Each treatment was defined by two levels of pruning and thinning. Data at single-tree level were collected to each sampling unit for estimating the most relevant parameter for the stand. Thirty-three trees in total were sampled. The growth strain was measured at the dbh in each one of them, with an extensometer designed by the CIRAD-Forêt. The pruning and thinning did not significantly affect the behavior of the growth strain in Eucalyptus globulus according
\end{abstract}

1 Ingeniero Forestal, MSc., Universidad de Concepción, Campus Los Ángeles, Escuela de Ciencias y Tecnologías, Departamento de Ciencias y Tecnología Vegetal. Grupo de Estudios Silvoecológicos (GESE). Juan Antonio Coloma 0201, Los Ángeles, Chile. ednavarr@udec.cl

2 Ingeniero Forestal, Profesional independiente, Juan Antonio Coloma 0201, Los Ángeles, Chile. cvalenzuela81@gmail.com

3 Ingeniero Forestal, Dr., Consultor Senior na Malinovski Florestal, Rua Itupava, 1541, CEP 80000, Curitiba (PR), lnutto.ufpr@gmail.com

Recebido para publicação em 7/02/2011 e aceito em 1/12/2014

Ci. Fl., v. 26, n. 3, jul.-set., 2016 
to the research study; on the other hand, the crown class had a highly significant affect on the variation of strains, showing the highest levels of it in dominant crown class trees that trees belonging to co-dominant and intermediate crown classes, respectively. The logarithmic functions and the variance obtained by measurements of dbh, crown radii, and pruning respectively, were entered into regression models as predictor variables of behavior of growth strain, showing a $\mathrm{R}^{2} 0,44$ and a SEE 0,132 .

Keywords: crown class; Eucalyptus; growth strain; silvicultural treatment.

\section{INTRODUCCIÓN}

El aumento en los últimos años de las plantaciones del género Eucalyptus en Chile, ha llevado a la necesidad de conocer de mejor forma las características de su madera (ROZAS Y GALLARDO, 2003). Su rápido crecimiento $\mathrm{y}$ adaptabilidad al clima y suelo del país, han permitido establecer plantaciones forestales que totalizan a la fecha 585.078 hectáreas, de las cuales cerca de dos tercios corresponden a Eucalyptus globulus Labill (LEANDRO et al., 2008).

Las especies del género Eucalyptus (en especial Eucalyptus globulus), han sido utilizadas en la fabricación de celulosa para la confección de papel y, en menor grado, en la fabricación de chapas, debido a la excelente apariencia de su veteado, en desmedro de la obtención de productos con mayor valor agregado (ROZAS Y GALLARDO, 2003). Esto por no poseer el conocimiento científico suficiente sobre los esquemas de manejo silvícola que tiendan a realzar la calidad de estos bosques, y con ello, aumentar su rentabilidad financiera (NEIRA, 2007).

El conocimiento de la variabilidad en las propiedades físicas y mecánicas es de fundamental importancia para los usuarios de la madera al momento de evaluar sus múltiples aplicaciones, y para los silvicultores, al momento de fijar los objetivos de producción en términos de manejo (DAVEL et al., 2005). Esto permite optimizar ciertas propiedades de acuerdo a las necesidades industriales y disminuir, dentro de ciertos límites, la heterogeneidad de la materia prima (ANANÍAS, 2008).

La utilización de la madera de Eucalyptus globulus no está exenta de dificultad, puesto que sus propiedades físicas y mecánicas están marcadas por una fuerte anisotropía y heterogeneidad, además de la presencia de tensiones internas de crecimiento, que son especialmente grandes en árboles jóvenes que tuvieron un desarrollo muy rápido (BAZO, 1999).
Durante el crecimiento del árbol, esfuerzos o tensiones internas se pueden desarrollar en su estructura. Estas tensiones son usualmente llamadas tensiones de crecimiento y se desarrollan en el cambium a medida que éste agrega una capa nueva de madera al tronco. Según Yang y Waugh (1996), las deformaciones residuales longitudinales (DRL), medidas en forma directa a través del método CIRAD-FORËT, pueden ser utilizadas como indicadores de las tensiones de crecimiento. Hoy en día no existe consenso sobre la causa exacta de estas tensiones, y cómo éstas son generadas (GACITÚA et al., 2007). La intensidad de las tensiones de crecimiento es muy variable entre especies, entre árboles adyacentes de la misma especie, en distintos lugares e incluso a diferentes alturas del mismo árbol (CHAFE, 1985; GERARD, 1994). Estas tensiones se manifiestan en el tronco al momento del volteo; en las trozas, como grietas en los extremos; durante el aserrío de las tablas, a través de alabeos y rajaduras en los extremos y corazón quebradizo, que posteriormente se traduce en desclasificación y disminución del aprovechamiento de la madera (ARCHER, 1986; SALES, 1986; YANG y WAUGH; 1996). Para el género Eucalyptus son frecuentes las especies con tensiones de crecimiento elevadas, tales como Eucalyptus grandis, Eucalyptus globulus, Eucalyptus saligna, entre otras (TOUZA, 2001).

Según Malan (1993) y Touza (2001) todavía es muy escaso el conocimiento respecto a las posibles relaciones existentes entre silvicultura y tensiones de crecimiento. Para algunos autores, las evidencias disponibles sugieren que las técnicas silvícolas no parecen ser efectivas en el control de los niveles de estas tensiones (MALAN, 1988). Sin embargo, y aunque no ha podido demostrarse de forma científica, otros autores consideran que podría minimizarse el nivel de tensiones de crecimiento, si se mantuviesen las condiciones de crecimiento y la distribución espacial de los árboles lo más uniforme posible a lo largo de su vida (KUBLER, 1988).

$\mathrm{Si}$ bien en Chile existen algunas experiencias, a nivel productivo, de diferentes 
técnicas de aserrío para aminorar los efectos de las tensiones de crecimiento en Eucalyptus, no existe información científica acerca de la relación entre la silvicultura y las tensiones generadas por la especie Eucalyptus globulus.

Por este motivo, el presente estudio tuvo como objetivo general evaluar el efecto de la poda y raleo sobre las deformaciones residuales longitudinales (indicadores de tensiones de crecimiento) en Eucalyptus globulus; proponiéndose como objetivo específico, analizar y modelar el efecto de diferentes niveles de intervención de poda y raleo, además de la clase de copa, sobre el comportamiento de las deformaciones residuales a nivel del diámetro a la altura de pecho (Dap).

\section{MATERIALES Y MÉTODOS}

\section{Área de estudio}

El ensayo se ubica en el predio Villucura, comuna de Santa Bárbara, propiedad de la Universidad de Concepción, en el sector precordillerano andino de la región del Bío-Bío, Chile ( $37^{\circ} 35^{\prime} \mathrm{S}$ y $\left.72^{\circ} 00^{\prime} \mathrm{O}\right)$, aproximadamente a 460 m.s.n.m. Presenta una topografía ondulada a quebrada, suelo perteneciente a la serie Santa Bárbara (textura arcillosa, compactada y con algunos sectores erosionados). El clima es templado, presentando una temperatura y precipitación media anual de $13,8^{\circ} \mathrm{C}$ y $1.389 \mathrm{~mm}$, respectivamente (BRITO, 2004).

\section{Descripción del lugar de estudio}

El estudio se realizó en una plantación de Eucalyptus globulus de doce años de edad, la cual fue intervenida a los dos años y medio de establecida, con el objetivo de evaluar la influencia del manejo silvícola en el crecimiento y dominancia de la especie. Los tratamientos analizados correspondieron a dos intensidades de poda, acompañadas por dos densidades de raleo, definiéndose un total de cuatro tratamientos (Tabla 1), con tres repeticiones (parcelas) cada uno. Cada parcela posee una superficie de 252 $\mathrm{m}^{2}(21 \mathrm{~m} \times 12 \mathrm{~m})$. En cada unidad experimental se seleccionaron tres árboles según clase de copa (dominante, codominante e intermedia) de acuerdo a la metodología descripta por Daniel et al. (1982).
TABLA 1: Descripción de los tratamientos aplicados.

TABLE 1: Description of the applied treatment.

\begin{tabular}{ccc}
\hline Poda (P) & \multicolumn{2}{c}{ Raleo (R) } \\
\hline & $\mathrm{R}_{1600}$ & $\mathrm{R}_{800}$ \\
$\mathrm{P}_{0 \%}$ & $\mathrm{P}_{0 \%} \mathrm{R}_{1600}$ & $\mathrm{P}_{0 \%} \mathrm{R}_{800}$ \\
$\mathrm{P}_{60 \%}$ & $\mathrm{P}_{60 \%} \mathrm{R}_{1600}$ & $\mathrm{P}_{60 \%} \mathrm{R}_{800}$ \\
\hline
\end{tabular}

Donde: Poda (porcentaje de altura podada); Raleo (densidad residual); $\mathrm{P}_{0 \%}$ : sin poda (testigo); $\mathrm{R}_{1600}: 1600$ árb $\cdot$ ha $^{-1}$ (testigo); $\mathrm{P}_{60 \%}:$ poda hasta un $60 \%$ de la altura total; $\mathrm{R}_{800}: 800$ árb $\cdot \mathrm{ha}^{-1}$.

\section{Diseño experimental}

Para conocer el efecto de las intervenciones silvícolas y la clase de copa en la variación de las deformaciones residuales longitudinales(DRL) de la madera deEucalyptus globulus a nivel del Dap, se evaluaron los siguientes factores con sus respectivos niveles:

\section{A: Factor Poda:}

Niveles:

$\mathrm{a}_{0}$ : sin poda (tratamiento testigo)

$a_{1}$ : poda $60 \%$ de la altura total

B: Factor Raleo:

Niveles:

$\mathrm{b}_{0}: 1600$ árb $\cdot \mathrm{ha}^{-1}$ (densidad inicial)

$\mathrm{b}_{1}: 800$ árb $\cdot \mathrm{ha}^{-1}$ (densidad residual)

C: Factor Clase de copa:

Niveles:

$\mathrm{c}_{0}$ : Dominante

$c_{1}$ : Codominante

$\mathrm{c}_{2}:$ Intermedia

El análisis de los datos se realizó como un experimento factorial de $2 \times 2 \times 3$, bajo un diseño completamente aleatorio, muestreándose un total de 36 árboles.

Las variables evaluadas fueron: Diámetro a la altura de pecho (Dap) $(\mathrm{cm})$, medido a $1,3 \mathrm{~m}$ desde el suelo; Altura total (m), determinada desde el suelo hasta el ápice principal; Clase de copa, determinada de acuerdo a la clasificación de Kraft en las categorías de árboles dominantes, codominantes e intermedios (DANIEL et al., 1982); Cobertura de copa (m), determinada desde el centro del árbol, a 
través de la medición de ocho radios según puntos cardinales y DRL (mm).

\section{Medición de tensiones}

Las medidas de las deformaciones residuales longitudinales (DRL) de maduración (indicador de tensiones de crecimiento) se realizaron según el método de "single hole drilling method" (según el protocolo de CIRAD-FORÊT) (TOUZA, 2001). El valor de las DRL se determinó a través de la medición por medio de un extensiómetro, en 4 puntos cardinales $(\mathrm{N}, \mathrm{S}, \mathrm{E}, \mathrm{O})$ sobre el perímetro descortezado del árbol. El extensiómetro propuesto por CIRAD-FORÊT permite evaluar en forma indirecta las DRL en el árbol en pie, y está basado en la teoría del agujero, que es aplicada para medir las tensiones residuales en la mayoría de los materiales, por cuanto al romper el equilibro mediante un agujero, las deformaciones provocadas por las fuerzas de tensión internas tienden a volver a su lugar de origen (DEVLIEGER y QUINTANA, 2006). Los estudios teóricos demuestran que las deformaciones se estabilizan a su valor máximo cuando la profundidad de perforación es equivalente al diámetro del agujero, y se capta la mayor deformación posible, cuando se realiza la medición a una distancia del centro del agujero igual a 1,73 veces el radio de éste. En forma práctica, se efectúa un orificio con una broca de $20 \mathrm{~mm}$ en el punto medio entre dos clavos de referencia, posicionados a una distancia entre sí de $45 \mathrm{~mm}$ en una paralela al eje de la médula del árbol. La medición del desplazamiento $(\delta)$ entre los clavos se realizó con un comparador digital con una precisión de $0,01 \mathrm{~mm}$. El valor registrado es un desplazamiento proporcional a las tensiones residuales longitudinales de maduración (T) que se relaciona a través de la expresión $\mathrm{T}=$ $\mathrm{k} \times \delta \times \mathrm{E}_{1}$ donde $\mathrm{k}$ es una variable que depende del diámetro de la broca, distancia entre los clavos, coeficiente de Poisson y $\mathrm{E}_{1}$ el módulo de elasticidad longitudinal de la madera (FOURNIER et al., 1994).

\section{Análisis estadístico}

El efecto de las distintas fuentes de variación (poda, raleo y clase de copa) sobre las DRL a nivel del Dap y sus interacciones, fue evaluado a través de un análisis de varianza (ANDEVA) por medio de prueba $\mathrm{F}$, y las diferencias detectadas identificadas por medio del test de Duncan, con un nivel de significancia de 0,05 (MONTGOMERY, 1991).
El modelo estadístico lineal que representa la variable de interés es el siguiente:

$$
\begin{aligned}
& Y_{i j k l}=\mu+P_{i}+R_{j}+C_{k}+(P R)_{i j}+(P C)_{i k}+(R C)_{j k}+ \\
& (P R C)_{i j k}+\varepsilon_{i j k l}
\end{aligned}
$$

Donde $\mathrm{Y}_{\mathrm{ijkl}}$ : Medida de las DRL; $\mu$ : Media poblacional; P: Poda (i): Efecto del i-ésimo nivel del factor Poda; R: Raleo (j): Efecto del j-ésimo nivel del factor Raleo; C: Clase de copa (k): Efecto del k-ésimo nivel del factor Clase de copa; (PR) ii: Efecto de la interacción poda y raleo; $(\mathrm{PC})_{\mathrm{ik}}$ : Efecto de la interacción poda y clase de copa; (RC) ${ }_{\mathrm{j} k}$ : Efecto de la interacción raleo y clase de copa; $(\mathrm{PRC})_{\mathrm{ijk}}$ : Efecto de la interacción poda, raleo y clase de copa y $\varepsilon_{\mathrm{ijkl}}:$ Error asociado al modelo.

El modelamiento de las DRL a nivel del Dap se realizó utilizando como variables predictivas las características tanto a nivel de árbol individual, como de rodal.

La estructura general del modelo de estimación de las DRL fue:

$$
D R L=f(\text { manejo, rodal y árbol })+\text { error }
$$

Donde Manejo: Poda $1=$ Con poda $(60 \%)$ y $0=$ Sin poda; Raleo $1=$ Con raleo $\left(800\right.$ árb $\left.\cdot h^{-1}\right)$ y 0 = Sin raleo (1600 árb·ha $\left.{ }^{-1}\right)$ (variables cualitativas); Atributos del rodal: variables de crecimiento (altura y diámetro promedio) y densidad (número de árboles y área basal por hectárea) y Características del árbol: Dap, altura total y clase de copa (variable cualitativa).

Con la información de las proyecciones de copa se realizaron perfiles horizontales (formación de poligonales), de modo de verificar la distribución y forma de las copas. Para analizar el efecto de la forma de la copa en el comportamiento de las deformaciones residuales, se calcularon las varianzas de los radios de copa, para luego relacionarlas con las DRL promedio por árbol.

$$
Y_{i}=X_{i} \beta+\varepsilon_{i}
$$

El modelo lineal para la expresión de las DRL a nivel del Dap en su forma matricial fue el siguiente:

Donde $\mathrm{Y}_{\mathrm{i}}=$ es un vector $\mathrm{N}_{\mathrm{i}} \times 1$ de observaciones para el i-ésimo árbol; $\beta=$ es un vector $\mathrm{p} \times 1 \mathrm{de}$ coeficientes de regresión; $\mathrm{p}=\mathrm{k}+1 ; \mathrm{X}_{\mathrm{i}}=$ es 
una matriz no estocástica $\mathrm{N}_{\mathrm{i}} \times \mathrm{p}$ de rango $\mathrm{p}$ de observaciones en las variables predictivas y $\varepsilon_{\mathrm{i}}=$ es un vector $\mathrm{N}_{\mathrm{i}} \times 1$ de errores aleatorios.

La estimación de parámetros se realizó a través de Mínimos Cuadrados Ordinarios (M.C.O.), y la selección de variables importantes en el modelo, por medio del método Forward Stepwise Regression, el cual se basa en la incorporación paso a paso de variables predictivas, que aporten en forma significativa a la variación de los residuos aún no explicadas por el modelo anterior (aumento del valor estadístico F de Snedecor) (MONTGOMERY, 1991). Finalmente, se realizó la evaluación de los modelos, analizando sus Coeficientes de determinación $\left(\mathrm{R}^{2}\right)$, Prueba $F$ de Snedecor para la regresión, Prueba $t$ de Student para los parámetros estimados y Error Estándar de Estimación (EEE). El cumplimiento de los supuestos de los errores de normalidad, homocedasticidad e independencia, se verificó a través de los test de Kolgomorov-Smirnov (K-S), Bartlett y Durbin-Watson (D-W) (Gujarati, 1992), respectivamente. Se realizaron transformaciones tanto a la variable dependiente como independientes en el modelo, con la finalidad de mejorar su bondad de ajuste y evitar multicolinealidad, comprobándose esta última, a través del factor de inflación de varianza (VIF) (Neter et al., 1996). Los análisis estadísticos se realizaron con el software Statistica 6.0 .

\section{RESULTADOS Y DISCUSIÓN}

\section{Caracterización dendrométrica de los tratamientos analizados}

El manejo silvícola no tuvo un efecto importante en las variables altura y diámetro promedio, no así en el área de copa, donde los tratamientos con raleo $\left(\mathrm{P}_{0 \%} \mathrm{R}_{800}\right.$ y $\left.\mathrm{P}_{60 \%} \mathrm{R}_{800}\right)$ presentaron los mayores valores para esta variable (9,13 y $9,84 \mathrm{~m}^{2}$, respectivamente). En cambio, para cada tratamiento silvicultural, las distintas clases de copa manifestaron diferencias tanto en altura, diámetro y área de copa promedio, siendo los individuos dominantes, los que presentaron los mayores valores para la totalidad de las variables evaluadas, seguidos por los codominantes e

TABLA 2: Descripción dendrométrica de los tratamientos estudiados.

TABLA 2: Dendrometric description of the studied treatments.

\begin{tabular}{|c|c|c|c|c|c|}
\hline \multicolumn{2}{|c|}{ Manejo silvícola } & \multirow[t]{2}{*}{ Clase de copa } & \multirow{2}{*}{$\begin{array}{c}\begin{array}{c}\text { Altura } \\
\text { promedio }\end{array} \\
(\mathrm{m})\end{array}$} & \multirow{2}{*}{$\frac{\begin{array}{c}\text { Diámetro } \\
\text { promedio }\end{array}}{(\mathrm{cm})}$} & \multirow{2}{*}{$\begin{array}{c}\text { Área de copa } \\
\text { promedio } \\
\left(\mathrm{m}^{2}\right)\end{array}$} \\
\hline Poda & Raleo & & & & \\
\hline \multirow{8}{*}{$\mathrm{P}_{0 \%}$} & \multirow{4}{*}{$\mathrm{R}_{1600}$} & Dominante (D) & $32,92(3,26)$ & $30,87(1,37)$ & $10,10(0,52)$ \\
\hline & & Codominante $(\mathrm{C})$ & $28,00(2,18)$ & $20,50(2,26)$ & $5,01(0,26)$ \\
\hline & & Intermedia (I) & $21,33(2,47)$ & $15,53(1,44)$ & $4,16(0,24)$ \\
\hline & & Promedio & 27,42 & 22,3 & 6,42 \\
\hline & \multirow{4}{*}{$\mathrm{R}_{800}$} & Dominante (D) & $29,17(4,16)$ & $33,17(2,30)$ & $16,72(0,62)$ \\
\hline & & Codominante $(\mathrm{C})$ & $24,83(3,79)$ & $19,67(2,31)$ & $6,71(0,47)$ \\
\hline & & Intermedia (I) & $15,67(7,01)$ & $11,53(3,13)$ & $3,97(0,47)$ \\
\hline & & Promedio & 23,22 & 21,46 & 9,13 \\
\hline \multirow{8}{*}{$\mathrm{P}_{60 \%}$} & \multirow{4}{*}{$\mathrm{R}_{1600}$} & Dominante (D) & $27,83(2,31)$ & $25,47(2,50)$ & $12,28(0,59)$ \\
\hline & & Codominante $(\mathrm{C})$ & $25,33(4,65)$ & $19,43(2,84)$ & $5,75(0,38)$ \\
\hline & & Intermedia (I) & $15,17(2,75)$ & $11,53(1,53)$ & $5,81(0,28)$ \\
\hline & & Promedio & 22,78 & 18,81 & 7,95 \\
\hline & \multirow{4}{*}{$\mathrm{R}_{800}$} & Dominante (D) & $27,50(4,44)$ & $27,47(4,65)$ & $14,39(0,74)$ \\
\hline & & Codominante $(\mathrm{C})$ & $27,50(4,09)$ & $24,00(2,29)$ & $8,46(0,61)$ \\
\hline & & Intermedia (I) & $21,33(7,97)$ & $15,60(5,93)$ & $6,68(0,52)$ \\
\hline & & Promedio & 25,44 & 22,36 & 9,84 \\
\hline
\end{tabular}

Em que: Valores en paréntesis corresponden a desviación estándar 
intermedios, respectivamente (Tabla 2).

\section{Efecto del manejo silvícola}

De la totalidad de factores evaluados, sólo la clase de copa tuvo un efecto altamente significativo sobre la variación de las DRL, no así las intervenciones de poda y raleo, lo cual podría atribuirse tanto al tiempo transcurrido desde la aplicación de la intervención silvícola (diez años), como así también a que dicha intervención fuese realizada sólo en una única oportunidad (Tabla 3).

TABLA 3: Análisis de varianza para los distintos factores estudiados.

TABLA 3: Variance analysis for the different studied factors.

\begin{tabular}{lccc}
\hline Fuente de variación & GL & F & Valor-p \\
\hline Poda $(\mathrm{P})$ & 1 & 3,19 & 0,086 \\
Raleo $(\mathrm{R})$ & 1 & 0,62 & 0,437 \\
Clase de Copa (C) & 2 & 5,59 & $<0,010$ \\
$\mathrm{P} \times \mathrm{R}$ & 1 & 0,17 & 0,682 \\
$\mathrm{P} \times \mathrm{C}$ & 2 & 1,68 & 0,208 \\
$\mathrm{R} \times \mathrm{C}$ & 2 & 0,18 & 0,837 \\
$\mathrm{P} \times \mathrm{R} \times \mathrm{C}$ & 2 & 0,24 & 0,787 \\
\hline
\end{tabular}

Contrario a estos resultados, Caniza et al. (2006) señalan que es posible modificar, controlar y minimizar, hasta cierto punto, el nivel de las tensiones, para esto es necesario limitar las provocaciones que creen incentivos por parte del árbol para competir. Esta limitación de estímulos está en su mayoría, relacionado principalmente con el manejo de la densidad del rodal y con los tratos silviculturales. Por su parte, Kubler (1988), asegura que es posible minimizar el nivel de tensiones, al mantener las condiciones de crecimiento y la distribución espacial de los árboles lo más uniforme posible a lo largo de su vida.

Los tratamientos con poda $\left(\mathrm{P}_{60^{\circ}}\right)$ presentaron un valor promedio de DRL mayor $(0,136 \mathrm{~mm})$ que aquellos tratamientos sin esta intervención $\left(\mathrm{P}_{0 \%}: 0,108 \mathrm{~mm}\right)$, aunque el efecto de este factor no resultó significativo a un nivel de $0,05(\mathrm{p}=$ 0,086). Esta misma situación se manifestó para los tratamientos con raleo $\left(\mathrm{R}_{800}\right)$, los cuales presentaron en promedio, un valor de DRL mayor $(0,128 \mathrm{~mm})$ a aquellos tratamientos sin intervención $\left(\mathrm{R}_{1600}: 0,116\right.$ $\mathrm{mm})$; sin embargo, estas diferencias no lograron ser estadísticamente significativas ( $\mathrm{p}=0,437)$.

Los valores máximo y mínimo en DRL se obtuvieron en los tratamientos con manejos extremos; es decir, $\mathrm{P}_{60 \%} \mathrm{R}_{800}(0,145 \mathrm{~mm})$ y $\mathrm{P}_{0 \%} \mathrm{R}_{1600}$ $(0,105 \mathrm{~mm})$, respectivamente, siendo el tratamiento de mayor intensidad de manejo, un 27,6\% superior al tratamiento testigo (sin manejo), aunque esta diferencia no fue estadísticamente significativa (Tabla 4).

Concordando con estos resultados, López et al. (2006), en un estudio para Eucalyptus grandis, evaluaron DRL a diferentes intensidades de raleo (1000 árb $\cdot$ ha $^{-1}, 700$ árb $\cdot h^{-1}, 500$ árb $\cdot h^{-1}, 300$ árb $\cdot h^{-1}$ y un tratamiento con dos raleos (500 árb $\cdot \mathrm{ha}^{-1}$ y 300 árb $\left.\cdot \mathrm{ha}^{-1}\right)$ ), determinando que estos no pudieron ser diferenciados estadísticamente. Similares resultados a los obtenidos en este estudio encontró Valdés (2004), al evaluar las DRL en 2 huertos semilleros de Eucalyptus nitens de 13 años de edad, ambos en la región del Bío Bío, Chile. El primer huerto con una densidad inicial de 1428 árboles/ha y densidad final de 438 árboles/ha $(3,5 \mathrm{~m} \times 2 \mathrm{~m})$, y el segundo, con una densidad inicial de 1667 árboles/ha y densidad final de 382 árboles/ha $(3 \mathrm{~m} \times 2 \mathrm{~m})$, presentando valores de DRL promedio que oscilaron entre 0,170 $\mathrm{mm}$ y $0,320 \mathrm{~mm}$, respectivamente, no siendo estos valores estadísticamente diferentes.

\section{Efecto de la clase de copa}

Independiente del tratamiento silvícola aplicado al rodal, las DRL promedio por clase de copa siempre fueron mayores en los árboles dominantes, seguido por los codominantes e intermedios, respectivamente (Figura 1). La clase de copa dominante presentó una DRL promedio de $0,150 \mathrm{~mm}$, superando en un $19 \%$ a la clase codominante $(0,126 \mathrm{~mm})$ y en un $70,4 \%$ a la clase intermedia $(0,088 \mathrm{~mm})$; presentándose para las clases de copa dominante e intermedia, diferencias estadísticamente significativas para el caso de los tratamientos con poda (Figura 1). Al respecto, y concordando con estos resultados, López (2002) en un estudio realizado para evaluar DRL en el estrato dominante para Eucalyptus grandis, determinó que a una misma edad de crecimiento, los individuos de mayor desarrollo en DAP, altura o volumen, también poseían un mayor nivel de deformaciones residuales. Por su parte, Biechele et al. (2008), al evaluar DRL en Eucalyptus nitens en diferentes 
TABLA 4: Valores de DRL promedio y variabilidad de copa según tratamiento silvícola y clase de copa. TABLE 4: Growth strains and crow variability values for silviculture treatment and crown class.

\begin{tabular}{|c|c|c|c|c|}
\hline \multicolumn{2}{|c|}{ Manejo silvícola } & \multirow{2}{*}{ Clase de copa } & \multirow{2}{*}{ DRL promedio $(\mathrm{mm})$} & \multirow{2}{*}{ DE radios de copa (m) } \\
\hline Poda & Raleo & & & \\
\hline \multirow{7}{*}{$\mathrm{P}_{0 \%}$} & \multirow{3}{*}{$\mathrm{R}_{1600}$} & Dominante (D) & $0,118 \mathrm{ab}$ & 0,524 \\
\hline & & Codominante $(\mathrm{C})$ & $0,114 \quad b$ & 0,259 \\
\hline & & Intermedia (I) & $0,084 \quad b$ & 0,242 \\
\hline & \multirow{5}{*}{$\mathrm{R}_{800}$} & Promedio & $0,105 a$ & 0,342 \\
\hline & & Dominante (D) & $0,118 \mathrm{ab}$ & 0,617 \\
\hline & & Codominante $(\mathrm{C})$ & $0,117 \mathrm{ab}$ & 0,467 \\
\hline & & Intermedia (I) & $0,097 \quad b$ & 0,473 \\
\hline \multirow{9}{*}{$\mathrm{P}_{60 \%}$} & & Promedio & $0,111 a$ & 0,519 \\
\hline & \multirow{3}{*}{$\mathrm{R}_{1600}$} & Dominante (D) & $0,161 \mathrm{ab}$ & 0,592 \\
\hline & & Codominante $(\mathrm{C})$ & $0,139 a b$ & 0,379 \\
\hline & & Intermedia (I) & $0,077 \mathrm{~b}$ & 0,280 \\
\hline & \multirow{5}{*}{$\mathrm{R}_{800}$} & Promedio & $0,126 a$ & 0,417 \\
\hline & & Dominante (D) & $0,204 \mathrm{a}$ & 0,740 \\
\hline & & Codominante (C) & $0,134 \mathrm{ab}$ & 0,607 \\
\hline & & Intermedia (I) & $0,096 \mathrm{~b}$ & 0,522 \\
\hline & & Promedio & $0,145 a$ & 0,623 \\
\hline
\end{tabular}

Letras iguales no difieren estadísticamente $(\mathrm{p}=0,05)$.

Con DRL = Deformación residual longitudinal; $\mathrm{DE}=$ Desviación estándar

etapas de desarrollo, determinaron que los árboles dominantes, debido a la mayor exposición al viento, presentaron una distribución de copas desequilibradas, lo que provoca mayor nivel de tensiones. No obstante, Touza (2001) en un estudio sobre sistemas de aserrado adecuados para procesar Eucalyptus globulus con tensiones de crecimiento (en plantaciones de 25 a 35 años), afirma que árboles dominantes presentan una distribución de tensiones reducida y equilibrada, debido a que al dominar en altura a los árboles que les rodean, carecen de estímulos para ascender en altura y/o reorientarse en una dirección concreta, por lo que pueden permitirse "relajar" su nivel de tensiones.

En la Tabla 4 y Figura 2 se observa que, independiente del tipo de manejo silvícola aplicado, existe una relación directamente proporcional entre las DRL promedio por clase de copa, y la asimetría presentada por éstas (expresada como desviación estándar de los radios de copa). En la Tabla 4, se observa además, que los árboles de los tratamientos de manejo extremos $\left(\mathrm{P}_{60 \%} \mathrm{R}_{800}\right.$ y $\mathrm{P}_{0 \%}$ $\mathrm{R}_{1600}$ ) presentaron los valores máximo y mínimo, tanto en DRL promedio $(0,145$ y $0,105 \mathrm{~mm})$, como en variabilidad de radios de copa $(0,623$ y $0,342 \mathrm{~m})$, respectivamente. La figura 2 muestra un ejemplo de unidades experimentales con condiciones extremas de manejo, en la cual se observa que la parcela de mayor intensidad de poda y raleo $\left(\mathrm{P}_{60 \%} \mathrm{R}_{800}\right)$, presenta copas de mayor superficie y asimetría que aquella sin manejo $\left(\mathrm{P}_{0 \%} \mathrm{R}_{1600}\right)$, lo que trae como consecuencia que se presenten, de igual manera, mayores DRL promedio en las primeras.

Al respecto, Delmastro et al. (1981) y Prado y Barros (1989), señalan que los efectos del espaciamiento y el raleo sobre las variables de un rodal son muy semejantes, ya que en ambos casos se afecta la disponibilidad de espacio de crecimiento de cada uno de los árboles, cambiando la estructura del rodal y favoreciendo además, el crecimiento de la copa. Barclay et al. (1986) señalan que el desarrollo de follaje depende principalmente de la penetración de luz y de la disponibilidad de nutrientes y agua, lo cual, según Muñoz et al. (2008) explicaría la mayor superficie foliar de los árboles presentes en tratamientos raleados. 


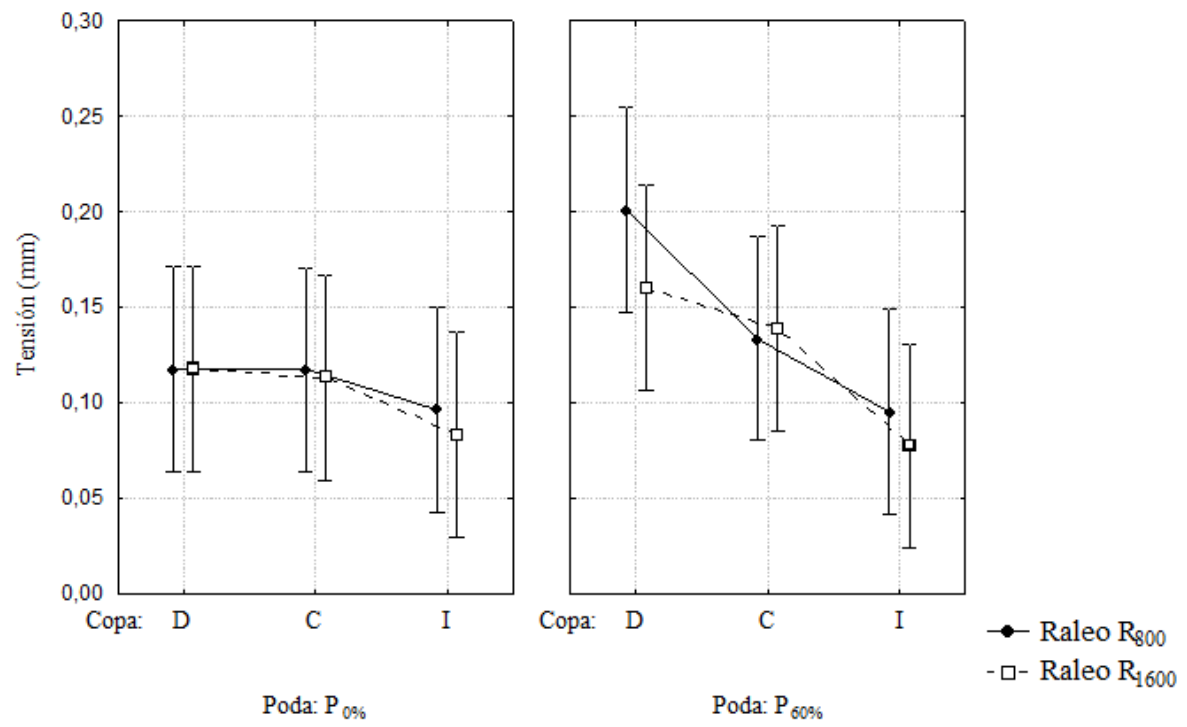

FIGURA 1: DRL y varianza de radios de copa según clase de copas y manejo silvícola.

FIGURE 1: Growth strain and crown radius variance for crown class and silviculture treatment.

Concordando con lo anterior, Maestri (2005) afirma que el espaciamiento disponible, a consecuencia de los raleos, permite mayor expansión de la copa, y el mayor peso de ésta somete al árbol a mayores esfuerzos. Según Schneider (1993), los troncos de las latifoliadas poseen una tendencia a inclinarse cuando el espacio disponible es amplio e irregular, siendo así, el control del espaciamiento tiene un efecto particularmente favorable sobre la inclinación, generando de esta manera, mayores tensiones en el tronco, situación que se observa en las tres clases de copas (árboles dominante, codominante e intermedio) de la parcela 12 . Similares resultados obtuvieron Caniza et al. (2006), en un estudio acerca de la variación de las DRL en tres clones de Eucalyptus grandis, según el estado de competencia individual, quienes determinaron un aumento en los valores de las tensiones con la intensidad del raleo. Sin embargo, estos resultados se contraponen a lo citado por Casado (1997), quien afirma que distintos espaciamientos entre árboles puede marcar la diferencia, aumentando los valores de las tensiones a mayor densidad de plantación. De igual manera, Nutto y Touza (2004), afirman que en espaciamientos densos, la elevada competencia impide el desarrollo de las copas, limitando el crecimiento diametral y generando DRL elevadas, concluyendo que las especies del género Eucalyptus, al crecer en condiciones de reducida competencia y con copas simétricas y bien desarrolladas, presentan menores deformaciones residuales.

El manejo de rodales, con la correcta aplicación de raleos que busquen dejar individuos distanciados simétricamente, permitirían reducir las provocaciones que generan la reorientación del fuste, de manera de aumentar sus rendimientos, controlando o minimizando los valores de las DRL (CANIZA et al., 2006). Este mismo autor considera que después de un raleo, y según la distribución espacial de los árboles, se pueden presentar dos situaciones o estados contrastantes de competencia: individuos que crecen manteniendo relativa simetría con sus "competidores" laterales más próximos, los que se encuentran distribuidos con cierta uniformidad a su alrededor; y otros, tienen a nuevos "competidores" laterales distribuidos asimétricamente a su alrededor, con algún vacío cercano producto del raleo de algunos árboles adyacentes.

Con la prudencia que debe acompañar siempre al género Eucalyptus, se permite interpretar el desarrollo de las DRL como una estrategia por parte de los árboles para competir de forma ventajosa por una posición privilegiada (TOUZA, 2001). Afirmación corroborada también Nutto y Touza (2004), quienes aducen que estudios recientes coinciden en señalar que es la competencia entre árboles y no su tasa de crecimiento la que genera deformaciones residuales elevadas. 


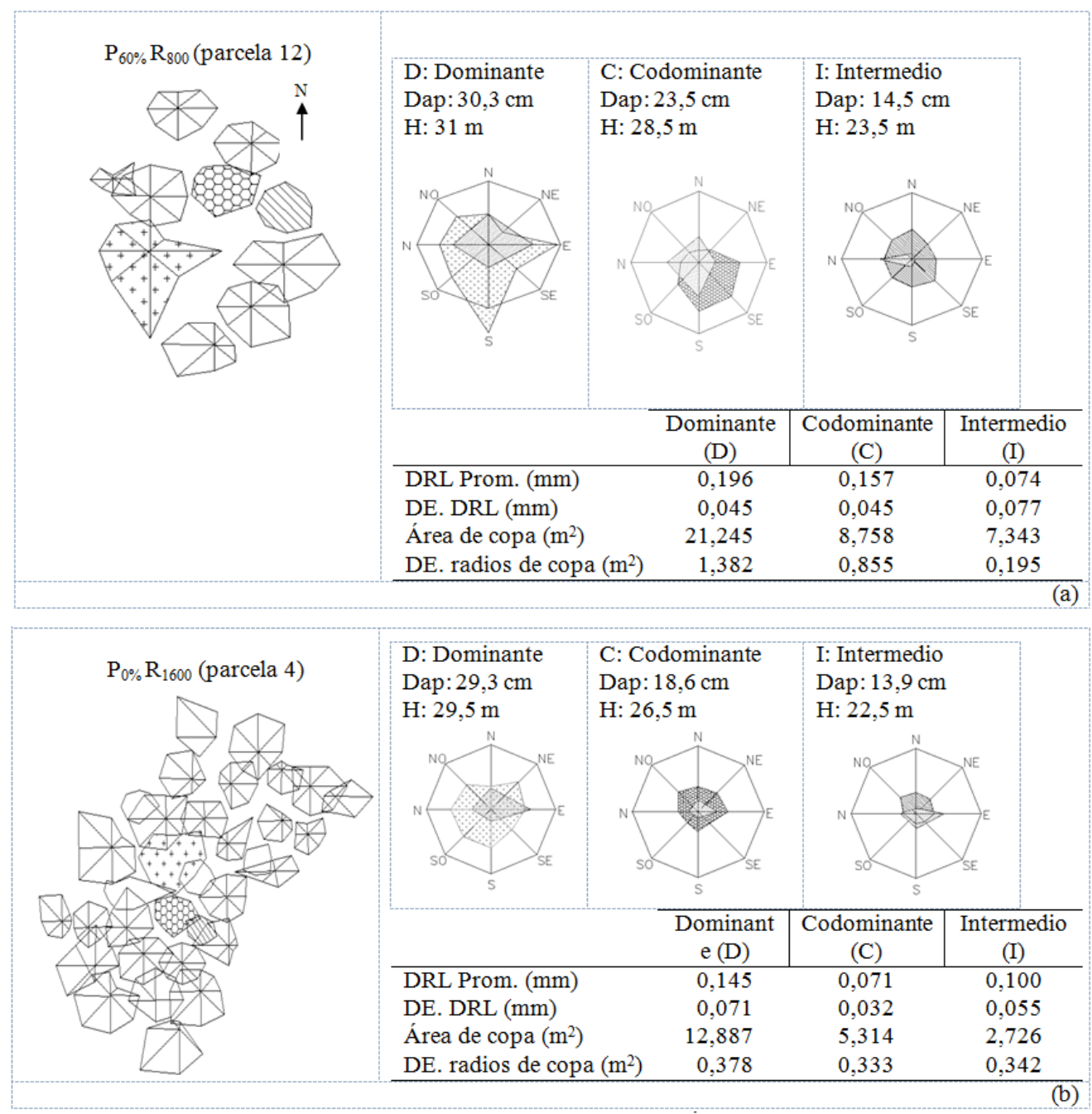

FIGURA 2: Perfil horizontal de dos parcelas con condiciones de manejo extremo (máxima (a) y sin intervención (b)); sus respectivos valores DRL promedios y desviación estándar (DE) en deformaciones residuales; distribución de las deformaciones en el fuste (achurado líneas finas), y la distribución y desviación de los radios de copa por punto cardinal (D: achurado cruces; C: achurado círculos; I: achurado líneas gruesas); además del Dap y la altura $(\mathrm{H})$ asociada a la clase de copa por árbol individual, según condición de manejo.

FIGURE 2: Horizontal profile of two plots with extreme management conditions (greatest (a) and without silviculture intervention (b)); the growth strain means and standard desviation (DE), fustal growth strain distribution (hatch fines lines), and cardinal point crown radius distribution and deviations (D: hatch crosses; C: hatch circles; I: hatch); management condition and crown class individual tree dbh and height $(\mathrm{H})$.

\section{Modelo de estimación}

El análisis de correlación muestra que sólo las variables dendrométricas Dap, altura total, área de copa y área basal presentaron significancia estadística con las DRL, no así las variables a nivel de rodal. Los valores positivos de los respectivos coeficientes demuestran una relación directamente proporcional entre las tensiones y el tamaño del árbol

Biechele et al. (2008), en un estudio para Eucalyptus nitens determinaron que a edades de entre 10 y 14 años, los factores que afectan el nivel de DRL son la altura y la posición social de los árboles. Por su parte, Valdés (2004) para Eucalyptus nitens sostiene que las tensiones están ligadas con el Dap; es decir, a mayor DAP las tensiones aumentarían, o al menos se mantendrían. Corroborando también los 
TABLA 5: Correlaciones entre DRL y variables dendrométricas y dasométricas.

TABLE 5: Correlations between dendrometric and dasometric values and growth strain

\begin{tabular}{lc}
\hline \multicolumn{1}{c}{ Variables } & DRL $(\mathrm{mm})$ \\
Altura total $(\mathrm{m})$ & $0,39 *$ \\
Dap $(\mathrm{cm})$ & $0,44^{*}$ \\
Coeficiente de esbeltez & $-0,28 \mathrm{~ns}$ \\
Área de copa $\left(\mathrm{m}^{2}\right)$ & $0,39 *$ \\
Varianza radios $\left(\mathrm{m}^{2}\right)$ & $0,19 \mathrm{~ns}$ \\
Área basal $\left(\mathrm{m}^{2} /\right.$ árb) & $0,37 *$ \\
Diámetro medio $(\mathrm{cm})$ & $0,07 \mathrm{~ns}$ \\
Altura media $(\mathrm{m})$ & $0,01 \mathrm{~ns}$ \\
Árb ha $^{-1}$ & $-0,22 \mathrm{~ns}$ \\
Área basal $\left(\mathrm{m}^{2} \cdot \mathrm{ha}^{-1}\right)$ & $-0,21 \mathrm{~ns}$ \\
\hline D́n
\end{tabular}

Donde: DRL: Deformación residual longitudinal; Dap: Diámetro a la altura de pecho (medido a 1,3 m sobre el nivel del suelo); Coeficiente de esbeltez (altura total dividida por el Dap); *: significativo ( $\mathrm{p}=0,05)$, ns: no significativo.

resultados obtenidos, López (2002) en Eucalyptus grandis, evidenció asociaciones positivas entre las DRL y $\mathrm{DAP}(\mathrm{R}=0,38)$, altura total $(\mathrm{R}=0,38)$ y volumen $(\mathrm{R}=0,37)$. Contrastando lo anterior, Vignote et al. (1996), señalan para Eucalyptus globulus, que la altura total del árbol apenas presenta o no influencia significativa con las DRL, y que el diámetro influye en menor medida sobre las tensiones.

En el modelo de DRL, las expresiones logarítmicas de las variables independientes Dap y Varianza de radios de copa (VR), además de la poda, fueron las que de mejor manera explicaron el modelo. Al aumentar los valores de DAP (transformado al logaritmo), la Varianza de los radios (inverso de la transformación logarítmica) y ante la presencia de poda, los valores de las DRL (transformadas al logaritmo) también aumentarían.
El modelo presentó un coeficiente de determinación $\left(\mathrm{R}^{2}=0,44\right)$, lo cual indica que la proporción de la variación de las DRL fue explicada en un $44 \%$ por el Log Dap, poda y $(1 /(\operatorname{LnVR}+1))$, presentando además, un error estándar de estimación de 0,132. Los valores de probabilidad (v-p) para los test de normalidad K-S y homocedasticidad de Bartlett fueron mayores al nivel de significación $(\mathrm{p}=0,05)$, demostrando el cumplimiento de estos supuestos. A su vez, el test de independencia D-W presentó un valor mayor al límite superior $\left(\mathrm{d}_{\mathrm{u}}=1,654\right)$ calculado para un tamaño de muestra de 36 y un número de variables predictoras igual a 3 , indicando la no correlación entre los residuos. El valor de inflación de la varianza (VIF) indica ausencia de multicolinealidad del modelo (VIF < 10) (NETER et al., 1996) (Tabla 6).

TABLA 6: Modelo de estimación de DRL.

TABLE 6: Growth strain estimation model.

\begin{tabular}{|c|c|c|c|c|c|c|c|c|}
\hline Modelo & $\mathrm{R}$ & $\mathrm{R}^{2}$ & EEE & $\begin{array}{l}\mathrm{K}-\mathrm{S} \\
\mathrm{v}-\mathrm{p}\end{array}$ & $\begin{array}{l}\text { Bartlett } \\
\text { v-p }\end{array}$ & D-W & VIF & $\mathrm{n}$ \\
\hline $\begin{array}{c}\log \mathrm{DRL}=-1,8203+0,6429 \log \mathrm{Dap}+0,0988 \\
\text { poda }-0,0164(1 /(\operatorname{LnVR}+1))+\varepsilon_{i}\end{array}$ & 0,66 & 0,44 & 0,13 & 0,76 & 0,94 & 1,95 & 1,1 & 36 \\
\hline
\end{tabular}

Donde: Log: Logaritmo en base 10, DRL: Deformaciones residuales longitudinales (mm), DAP: Diámetro altura de pecho $(\mathrm{cm})$, Poda: Variable cualitativa (1: con poda y 0: sin poda), VR: Varianza de los radios de copa $\left(\mathrm{m}^{2}\right), \varepsilon_{\mathrm{i}}$ : Error asociado al modelo, R: Coeficiente de correlación, $\mathrm{R}^{2}$ : Coeficiente de determinación, EEE: Error estándar de estimación, K-S v-p: valor de probabilidad test de normalidad de Kolmogorov-Smirnov; Bartlett v-p: valor de probabilidad test de homocedasticidad de Bartlett; D-W: valor estadístico test de independencia de Durbin y Watson; VIF: valor de factor de inflación de la varianza; n: Número de muestras. 


\section{CONCLUSIONES}

De acuerdo a las condiciones en las que se realizó el presente estudio, y considerando los resultados, se concluye que:

La poda y raleo no afectaron significativamente el comportamiento de las DRL en Eucalyptus globulus, aunque se presentaron mayores niveles de deformación residual en aquellos árboles sometido a intervenciones de mayor intensidad.

La clase de copa tuvo un efecto altamente significativo sobre la variación de las DRL, presentando los árboles dominantes mayores niveles de deformación residual que la clase de copa codominante e intermedia, respectivamente.

Independiente del tipo de manejo silvícola aplicado, existió una relación directamente proporcional entre las DRL promedio por clase de copa, y la asimetría presentada por éstas.

Las DRL fueron afectadas sólo por las variables dendrométricas Dap, altura total, área de copa y área basal, existiendo una relación directamente proporcional entre éstas y la variable respuesta; es decir, a medida que aumentaron los valores en estas variables, aumentó también el nivel de deformación a nivel individual.

El modelo de estimación de DRL incluyó como variables predictivas, las expresiones logarítmicas del Dap, varianza de los radios de copa; además de la poda.

\section{REFERENCIAS BIBLIOGRÁFICAS}

ANANÍAS, R. Física de la madera. Sin fecha de publicación. Disponible en: http://zeus.dci.ubiobio. $\mathrm{cl} /$ ananias/apuntes_fisica_madera.pdf. Consultado el 22 de Junio del 2008.

ARCHER, R. Growth stresses and strains in trees. Springer-Verlag, Berlin, 1986. 240 p.

BARCLAY, H.; PANG, P.; POLLARD, F. Aboveground biomass distribution within trees and stand in thinned and fertilized Douglas-fir. Can. J. For. Res., v. 16, p. 438-442, 1986.

BAZO, C. Usos alternativos de la madera de Eucalipto. Perspectivas de utilización en carpintería y mobiliario. Boletín de información técnica AITIM, v. 198, p. 19-22, 1999.

BIECHELE, T.; NUTTO, L.; NAVARRETE, E. Growth stress in Eucalyptus nitens at different stages of development. In: SOCIETY OF WOOD AND TECHNOLOGY (SWST) ANNUAL CONVENTION. Organizado por Society of Wood and Technology y la Universidad del Bío Bío. Noviembre 10 al 12, 2008. Concepción, Chile.

BRITO, C. Efecto de la poda y raleo en el crecimiento y dominancia de un rodal joven de Eucalyptus nitens en Chile. 2004. 60 p. Seminario de título de Ingeniería Ejecución Forestal. Universidad de Concepción. Los Ángeles, Chile.

CANIZA, F. et al. Variación de las tensiones de crecimiento en tres clones de Eucalyptus grandis según el estado de competencia individual. XXII JORNADAS FORESTALES DE ENTRE RÍOS, 2006, Concordia, Argentina.

CASADO, $M$. Tensiones de crecimiento en cinco clones de Populus $\boldsymbol{x}$ euroamericano; I-Ingenieros de Montes, Universidad politécnica de Madrid, Madrid, 1997. $215 \mathrm{p}$.

CHAFE, S. Variation in longitudinal growth stress with heigth in trees of Eucalyptus nitens Maiden. Australian Forest Research, v 15, n. 1, p. 51-55, 1985.

DANIEL, P.; HELMS, U.; BAKER, F. Principios de Silvicultura. Segunda ed. McGraw-Hill, México. 1982. $492 \mathrm{p}$.

DAVEL, M.; JOVANOVSKI, A.; MOHR BELL, D. Densidad básica de la madera de pino Oregón y su relación con las condiciones de crecimiento en la Patagonia Andina Argentina. Bosque (Valdivia), v. 26, n. 3, p. 55-62, 2005.

DELMASTRO, R.; DIAZ-VAZ, J.; SCHLATTER, J. Variabilidad de las características tecnológicas hereditarias del Pinus radiata (D. Don). Informe $\mathrm{N}^{\circ}$ 3. Chile. Universidad Austral de Chile, Chile, $1981.187 \mathrm{p}$.

DEVLIEGER, F.; QUINTANA, R. Tensiones de crecimiento en híbridos de Álamo creciendo en Chile. Maderas: Ciencia y Tecnología, v. 8, n. 3, p. 219-222, 2006.

FOURNIER, M. et al. Mesures des déformations résiduelles de croissance à la surface des arbres, en relation avec leur morphologie. Observations sur différentes espèces. Annals of Science Forestry, v.51, n. 3, p. 249-266, 1994.

GACITÚA, W. et al. Nanoidentaciones y ultraestructura en madera de Eucalyptus nitens con micro y meso grietas. Maderas: Ciencia y Tecnología, v. 9, n. 3, p. 259-270, 2007.

GERARD, M. Contraintes de Croissance, Variations Internes de Densite et de Module D'elasticite Longitudinal, et Deformations de Sciage Chez les Eucalyptus de Plantation. These pour Obtenir le Grade de Docteur L'Universite de Bordeaux I. Nº d'ordre: 1253, 1994. 160 p. 
GUJARATI, D. Econometría. Segunda edición, McGraw-Hill Interamericana, México. 597 p. 1996. KUBLER, H. Silvicultural control of mechanical stresses in trees. Canadian Journal of Forest Research, v. 18, n. 10, p. 1215-1225, 1988.

LEANDRO, L. etal. Estudio preliminar de las grietas internas en anillos de madera inicial y su relación con características de la estructura anatómica y densidad en Eucalyptus nitens. Interciencia, v. 33, n. 11, p. 829-834, 2008.

LÓPEZ, J. Tensiones de Crecimiento del Estrato Dominante de Algunos Orígenes y Procedencias de Eucalyptus grandis a los 17 Años de Edad en 2 Sitios del Oeste de la Provincia de Corrientes, Argentina. In: JORNADAS FORESTALES DE ENTRE RÍOS, 2002, Concordia, Argentina.

LÓPEZ, J.; APARICIO, J.; GENES, P. Efecto de Diferentes Intensidades de Raleo en la Densidad de la Madera y en las Tensiones de Crecimiento de Eucalyptus grandis. In: JORNADA FORESTAL EA. SAN ANTONIO, 2006, Corrientes, Argentina. MAESTRI, R. Ganhos Tecnológicos na Produçao de Maderira Reflorestada Sob Manejo. In: III SEMINARIO DE PRODUTOS SOLIDOS DE MADEIRA DE EUCALIPTO - SIF, 2005, Porto Alegre, Brasil.

MALAN, F. The control of end splitting in sawlogs; A short literatura review. South African Forestry Journal, v. 109, p. 14-18, 1988.

MALAN, F. The Wood Properties and Qualities of Three South African-grown Eucalypt Hybrids. South African Forestry Journal, v. 167, p. 35-44, 1993.

MONTGOMERY, D. Diseño y Análisis de Experimentos. Editorial Iberoamericana. México D.F., México, 1991. 589 p.

MUÑOZ, F. et al. Efecto de poda y raleo en el área foliar de Eucalyptus nitens. Bosque (Valdivia), v. 29, n. 1, p. 44-51, 2008.

NEIRA, A. Efecto del raleo en la densidad básica de la madera de Eucalyptus nitens en una plantación de 15 años ubicada en la provincia de Arauco. 2007. 39 p. Memoria de título de Ingeniería Forestal. Universidad de Concepción. Concepción,
Chile.

NETER, J. et al. Applied Linear Statistical Models. McGraw-Hill Companies, Third Edition, Irwin. 720 p. 1996.

NUTTO, L.; TOUZA, M. Producción de madera de sierra de alta calidad con Eucalyptus globulus. CIS-Madera: Revista del Centro de Innovación y Servicios Tecnológicos de la Madera, ISSN 1138-7726, v. 12, p. 6-18, 2004.

PRADO, J.; BARROS, S. Eucalyptus principios de silvicultura y manejo. INFOR/CORFO. Santiago, Chile, 1989. 199 p.

ROZAS, C.; GALLARDO, M. Tensiones de crecimiento y aprovechamiento en madera de Eucalyptus nitens y Eucalyptus globulus. En: Proyecto FONDEF. Pp: 28-55. FONDEFCONYCIT. Santiago, Chile, 2003.108 p.

SALES, C. Growth stresses: Main results obtained on the study of some Guyana species and consequences for end use. In: IUFRO WORLD CONGRESS (18.: Ljubljana). Proccedings. Blacksburg, VA: IUFRO 5, 1986. p. 234-248.

SCHNEIDER, P. Introdução ao Manejo Florestal. Santa Maria. RS: UFSM/CEPEF/FATEC, 1993. $348 \mathrm{p}$.

TOUZA, M. Proyecto de investigación sobre sistemas de aserrado adecuados para procesar Eucalyptus globulus con tensiones de crecimiento. CIS-Madera, v. 6, p. 8-37, 2001.

VALDÉS, R. Tensiones de crecimiento en Eucalyptus nitens (Deane \& Maiden) mediante un método no destructivo. 2004. 61 p. Memoria de titulación. Universidad de Talca. Facultad de Ciencias Forestales. Chile.

VIGNOTE, S. et al. Estudio de las tensiones de crecimiento de Eucalyptus globulus Labill en Galicia y su relación con las características de la estación y morfológicas del propio árbol. Investigación Agraria. Sistemas y Recursos Forestales, v. 5, n. 1, p.153-165, 1996.

YANG, J.; WAUGH, G. Potential of plantationgrown eucalypts for structural sawn products. II. Eucalyptus nitens (Dean \& Maiden) Maiden and E. regans F. Muell. Aust Forest, v. 59, n. 2, p. 99-107, 1996. 